\title{
Some Properties of a Class of Band Matrices
}

\author{
By W. D. Hoskins and P. J. Ponzo
}

\begin{abstract}
Let $A(2 r+1, n)$ denote the $n \times n$ band matrix, of bandwidth $2 r+1$, with the binomial coefficients in the expansion of $(x-1)^{2 r}$ as the elements in each row and column. Using the fact that the rows of $A(2 r+1, n)$ provide the coefficients for the $2 r$ th central difference, a number of properties of $A(2 r+1, n)$ are obtained for all positive integers $r$ and $n$. These include obtaining explicit formulas for $\operatorname{det} A(2 r+1, n), A^{-1}(2 r+1, n)$, $\left\|A^{-1}(2 r+1, n)\right\|_{\infty}$ and an upper triangular matrix $U$ such that $A(2 r+1, n) U$ is lower triangular.
\end{abstract}

1. Introduction. We consider the set of band-diagonal matrices of bandwidth $2 r+1$, with the binomial coefficients in the expansion of $(x-1)^{2 r}$ displayed symmetrically about the diagonal in each row and column. If $A(2 r+1, n)$ denotes the $n$th order member of this set, then, for example

$$
A(5, n)=\left[\begin{array}{rrrrr}
6 & -4 & 1 & & \\
-4 & 6 & -4 & 1 & \\
1 & -4 & 6 & -4 & 1 \\
& & & . & \\
& & 1 & -4 & 6
\end{array}\right] .
$$

Such matrices occur quite frequently in a variety of contexts, [1]-[3]. It is known [4], for the tridiagonal matrix, that

$$
\operatorname{det}|A(3, n)|=n+1
$$

and

$$
\left\|A^{-1}(3, n)\right\|_{\infty} \leqq(n+1)^{2} / 8,
$$

with equality when $n$ is odd. We will obtain, below, these and other properties of $A(2 r+1, n)$, for general $r$.

2. In what follows, we use $A$ to denote $A(2 r+1, n)$ unless otherwise specified.

If $x$ is an arbitrary $n$-vector with components $x(i)(i=1(1) n)$, then we may extend the range of $i$ and define $x(i)=0$ for $i=0,-1,-2, \cdots,-r+1$ and for $i=n+1$, $n+2, \cdots, n+r$. With this extension, $A x$ is an $n$-vector whose components are precisely $\delta^{2 r} x(i)$, for $i=1(1) n$, since the rows of $A$ provide the coefficients for the $2 r$ th central difference. We use this notion to transform $A$ to a lower triangular matrix.

Received September 20, 1971

AMS 1969 subject classifications. Primary 6535; Secondary 6562.

Key words and phrases. Determinants, band matrices, inverse matrices, norms on inverses.

Copyright $\odot$ 1972, American Mathematical Society 
We consider, for any integer $j \geqq 1$,

(1)

$$
\begin{aligned}
\phi_{i}(i) & =\frac{i(i+1) \cdots(i+r-1)}{r !} \frac{(j+1-i)(j+2-i) \cdots(j+r-1-i)}{(r-1) !} \\
& =\left(\begin{array}{c}
i+r-1 \\
r
\end{array}\right)\left(\begin{array}{c}
j+r-1-i \\
r-1
\end{array}\right) .
\end{aligned}
$$

Then, $\phi_{i}(i)$ is a polynomial of degree $2 r-1$ in the variable $i$, with $\phi_{i}(i)=0$ for $i=$ $0,-1, \cdots,-r+1$ and $i=j+1, j+2, \cdots, j+r-1$.

We define an $n$-vector $u_{i}$ with components

$$
\begin{aligned}
u_{i}(i) & =\phi_{i}(i) & & \text { for } i<j+r, \\
& =0 & & \text { for } i \geqq j+r .
\end{aligned}
$$

Then, $u_{i}(i)$ is the polynomial $\phi_{i}(i)$ (of degree $2 r-1$ ) for $i<j+r$, so that $\delta^{2 r} u_{i}(i)$ $=0$ for $i=1,2, \cdots, j-1$. Hence, $A u_{j}$ has nonzero components only for $i=j$, $j+1, \cdots, n$. If $U$ denotes the upper triangular matrix whose $j$ th column is $u_{i}$, as given by (2), then $A U=L$ is lower triangular. We summarize in

THEOREM 1. If $A(2 r+1, n)=\left\{a_{i j}\right\}$ where

$$
a_{i j}=(-1)^{r+i-j}\left(\begin{array}{c}
2 r \\
r+i-j
\end{array}\right) \text {, }
$$

and $U=\left\{u_{i}\right\}$ where

$$
\begin{aligned}
u_{i i} & =\left(\begin{array}{rl}
i+r-1 \\
r
\end{array}\right)\left(\begin{array}{rl}
j+r-1-i \\
r-1
\end{array}\right) & \text { for } i \leqq j, \\
& =0 & \text { for } i>j,
\end{aligned}
$$

then $A U=L$ with $L$ lower triangular.

We will need the following lemmas.

LEMMA 1. If $A U=L$ and

(i) $A$ is an arbitrary Hermitian matrix (i.e. $A^{*}=A$ where $A^{*}$ is the conjugate transpose),

(ii) $U$ is upper triangular,

(iii) $L$ is lower triangular, then $L^{*} U=D$, where $D$ is a real, diagonal matrix.

The proof follows immediately on observing that any Hermitian matrix can be factorized into the form $R^{*} \tilde{D} R$ with $\tilde{D}$ a diagonal matrix.

LEMMA 2. If $L$ and $U$ are the matrices of Theorem 1 , then $L^{T} U=D$, a real diagonal matrix with

$$
D_{k k}=(-1)^{r}\left(\begin{array}{c}
k+2 r-1 \\
r
\end{array}\right)\left(\begin{array}{c}
k+r-1 \\
r
\end{array}\right)=(-1)^{r}\left(\begin{array}{c}
k+2 r-1 \\
2 r
\end{array}\right)\left(\begin{array}{l}
2 r \\
r
\end{array}\right) .
$$

Proof. Since $D=L^{T} U$, from Theorem 1 and Lemma 1 , then $D_{k k}=l_{k k} u_{k k}$. From (2), we have $u_{k k}=\phi_{k}(k)$ and, from Theorem $1, l_{k k}=\delta^{2 r} u_{k k}=-\phi_{k}(k+r)$. Substituting for $\phi$, as given by (1), we obtain 


$$
\begin{aligned}
D_{k k} & =(-1)\left(\begin{array}{c}
k+r-1 \\
r
\end{array}\right)\left(\begin{array}{c}
k+2 r-1 \\
r
\end{array}\right)\left(\begin{array}{c}
-1 \\
r-1
\end{array}\right) \\
& =(-1)^{r}\left(\begin{array}{c}
k+r-1 \\
r
\end{array}\right)\left(\begin{array}{c}
k+2 r-1 \\
r
\end{array}\right),
\end{aligned}
$$

where $\left(\begin{array}{c}-1 \\ r-1\end{array}\right)=(-1)^{r-1}$. This expression for $D_{k k}$ can be written more simply as

$$
D_{k k}=(-1)^{r}\left(\begin{array}{c}
k+2 r-1 \\
2 r
\end{array}\right)\left(\begin{array}{l}
2 r \\
r
\end{array}\right) \text {. }
$$

THEOREM 2.

$$
\operatorname{det} A(2 r+1, n)=(-1)^{n} \prod_{k=1}^{n}\left\{\frac{\phi_{k}(k+r)}{\phi_{k}(k)}\right\} \text {. }
$$

Proof. From $A U=L$, we obtain

$$
\operatorname{det} A=\operatorname{det} L / \operatorname{det} U=\prod_{k=1}^{n} l_{k k} / \prod_{k=1}^{n} u_{k k} .
$$

Substituting $u_{k k}=\phi_{k}(k)$ and $l_{k k}=-\phi_{k}(k+r)$ gives the required result.

The above expression for $\operatorname{det} A$ simplifies considerably. It is perhaps more revealing to write it out for the first few values of $r$.

$$
\begin{aligned}
& \operatorname{det} A(3, n)=n+1 \\
& \operatorname{det} A(5, n)=\frac{(n+1)(n+2)^{2}(n+3)}{1 \cdot 2^{2} \cdot 3} \\
& \operatorname{det} A(7, n)=\frac{(n+1)(n+2)^{2}}{1 \cdot 2^{2} \cdot} \cdot \frac{(n+3)^{3}(n+4)^{2}(n+5)}{3^{3} \cdot 4^{2} \cdot 5}
\end{aligned}
$$

In general, det $A(2 r+1, n)$ is a polynomial in $n$, of degree $r^{2}$, with zeros at $n=$ $-1,-2, \cdots,-2 r+1$.

We turn now to the computation of $\left\|A^{-1}\right\|_{\infty}$. We will need the following lemmas.

LEMMA 3. If the conditions of Lemma 1 are satisfied, then $A^{-1}=U D^{-1} U^{*}$, if the inverse exists.

Proof. From Lemma $1, A U=L=\left(U^{*}\right)^{-1} D$. Hence, $A=\left(U^{*}\right)^{-1} D U^{-1}$ and $A^{-1}=$ $U D^{-1} U^{*}$.

LEMMA 4. If e denotes the $n$-vector, all of whose components are unity, and $D, U$ are the matrices of Lemma 2, then $e$ is an eigenvector of $D^{-1} U^{*}$. More specifically, $D^{-1} U^{*} e$ $=\lambda e$ with $\lambda=(-1)^{r} /\left(\begin{array}{c}2 r \\ r\end{array}\right)$.

Proof. The $k$ th component of $D^{-1} U^{*} e$ is

$$
\left(\frac{1}{D_{k k}}\right) \sum_{i=1}^{k} \phi_{k}(j)=\left(\frac{1}{D_{k k}}\right) \sum_{i=1}^{k}\left(\begin{array}{c}
j+r-1 \\
r
\end{array}\right)\left(\begin{array}{c}
k+r-1-j \\
r-1
\end{array}\right) \text {, }
$$

where the summation is the $k$ th column sum of $U$.

From [5], we have the identity 


$$
\sum_{j=1}^{k-1}\left(\begin{array}{c}
k+r-j-2 \\
k-j-1
\end{array}\right)\left(\begin{array}{c}
j+r \\
j
\end{array}\right)=\left(\begin{array}{c}
k+2 r-1 \\
2 r
\end{array}\right) .
$$

Using (4), slightly modified, we may sum the series in (3) to obtain the $k$ th component in the form $\left(1 / D_{k k}\right)\left({ }_{2 r}^{k+2 r-1}\right)$. Substituting for $D_{k k}$, from Lemma 2, gives $(-1)^{r} /\left(\begin{array}{c}2 r \\ r\end{array}\right)$, which, for $k=1(1) n$, is just the $k$ th component of $\lambda e$, with $\lambda=(-1)^{r} /\left(\begin{array}{c}2 r \\ r\end{array}\right)$. This establishes the result.

THEOREM 3. The row-sums of $A^{-1}$ are given by

$$
S_{i}=(-1)^{r} \frac{\left(\begin{array}{c}
j+r-1 \\
r
\end{array}\right)\left(\begin{array}{c}
n-j+r \\
r
\end{array}\right)}{\left(\begin{array}{c}
2 r \\
r
\end{array}\right)} \text { for } j=1(1) n .
$$

Proof. If $\hat{e}_{i}$ denotes the unit $n$-vector with 1 in the $j$ th position and, as in Lemma $4, e=\hat{e}_{1}+\hat{e}_{2}+\cdots+\hat{e}_{n}$, then the $j$ th row-sum of $A^{-1}$ can be written as $S_{i}=$ $\left(\hat{e}_{i}\right)^{T} A^{-1} e$.

Substituting for $A^{-1}$ from Lemma 3 (and using Lemma 4) gives $S_{i}=\lambda\left(\hat{e}_{i}\right)^{T} U e$ where $\lambda=(-1)^{r} /\left(\begin{array}{c}2 r \\ r\end{array}\right)$. But $\left(\hat{e}_{i}\right)^{T} U e$ is just the jth row-sum of $U$, which is $\sum_{i=i}^{n} \phi_{i}(j)$. Substituting for $\phi_{i}(j)$ gives

$$
S_{j}=\frac{(-1)^{r}}{\left(\begin{array}{c}
2 r \\
r
\end{array}\right)}\left(\begin{array}{c}
j+r-1 \\
r
\end{array}\right) \sum_{i=j}^{n}\left(\begin{array}{c}
i-j+r-1 \\
r-1
\end{array}\right) \quad \text { for } j=1(1) n .
$$

It is a simple matter to show, by induction on $n$, that

$$
\sum_{i=j}^{n}\left(\begin{array}{c}
i-j+r-1 \\
r-1
\end{array}\right)=\left(\begin{array}{c}
n-j+r \\
r
\end{array}\right),
$$

which gives the required result.

THEOREM 4.

$$
\begin{aligned}
\left\|A^{-1}(2 r+1, n)\right\|_{\infty} & =\frac{\prod_{k=1}^{r}(n+2 k-1)^{2}}{2^{2 r}(2 r) !} \text { for } n \text { odd }, \\
& =\frac{\prod_{k=1}^{r}(n+2 k-2)^{2}}{2^{2 r}(2 r) !}\left(\frac{n+2 r}{n}\right) \text { for } n \text { even. }
\end{aligned}
$$

Proof. From Theorem 3 and the definition of the infinity-norm, $\left\|A^{-1}\right\|_{\infty}=$ $\max _{i}\left|S_{j}\right|$.

But $S_{i}$ is a polynomial in $j$, of degree $2 r$, with zeros at $j=0,-1, \cdots,-r+1$ and $j=n+1, n+2, \cdots, n+r$. Application of Rolle's theorem guarantees that $d S_{i} / d j$ vanishes just once between successive zeros. In particular, $d S_{i} / d j$ vanishes precisely once in $0<j<n+1$. However, $S_{i}$ is symmetric about $j=(n+1) / 2$, since $S_{n+1-i}=S_{i}$. Hence, for $0<j<n+1,\left|S_{i}\right|$ attains an absolute maximum at $j=$ $(n+1) / 2$. If $(n+1) / 2$ is an integer (i.e., $n$ is odd), then $\left\|A^{-1}\right\|_{\infty}=\left|S_{i}\right|$ with $j=$ $(n+1) / 2$. If $n$ is even, then $\left\|A^{-1}\right\|_{\infty}=\left|S_{i}\right|$ with $j=n / 2$ (or $\left.1+n / 2\right)$. Hence,

$$
\begin{aligned}
& \left\|A^{-1}\right\|_{\infty}=\left(\begin{array}{c}
(n-1) / 2+r \\
r
\end{array}\right)^{2} /\left(\begin{array}{c}
2 r \\
r
\end{array}\right) \text { for } n \text { odd, } \\
& \left\|A^{-1}\right\|_{\infty}=\left(\begin{array}{c}
n / 2+r-1 \\
r
\end{array}\right)\left(\begin{array}{c}
n / 2+r \\
r
\end{array}\right) /\left(\begin{array}{l}
2 r \\
r
\end{array}\right) \text { for } n \text { even. }
\end{aligned}
$$


Expanding the binomial coefficients and rearranging somewhat gives the statement of the theorem.

We turn to the problem of determining $A^{-1}(2 r+1, n)$.

From Lemma 3, we have $A^{-1}=U D^{-1} U^{T}$ so that

$$
\left\{A^{-1}\right\}_{i i}=\sum_{k=1}^{n} \frac{u_{i k} u_{i k}}{D_{k k}}
$$

Substituting for $u$ (from Theorem 1 ) and $D_{k k}$ (from Lemma 2) gives, for $i \geqq j$, LEMMA 5.

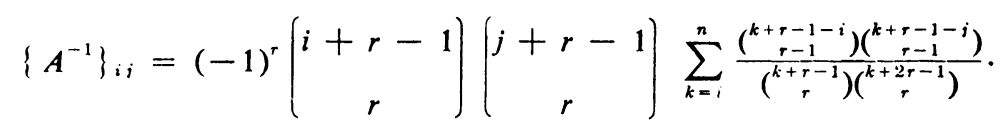

Here, we have obtained the elements below, and on the main diagonal. The remaining elements of $A^{-1}$ are obtained by symmetry.

It is instructive to obtain $A^{-1}$ somewhat differently, as follows.

Let

$$
p(x)=\frac{x\left(x^{2}-1^{2}\right)\left(x^{2}-2^{2}\right) \cdots\left[x^{2}-(r-1)^{2}\right]}{r\left(r^{2}-1^{2}\right) \cdots\left[r^{2}-(r-1)^{2}\right]},
$$

so that $p(x)=0$ at $x=0, \pm 1, \pm 2, \cdots, \pm(r-1)$, and $p( \pm r)= \pm 1$. If $x$ is an integer, we also have

$$
p(x)=\left(\begin{array}{c}
x+r-1 \\
2 r-1
\end{array}\right)
$$

We now define, for any $j \geqq 1$, the (unique) polynomial in $i$ of degree $(2 r-1)$, $f_{i}(i)$, which satisfies

$$
\begin{aligned}
& \text { (a) } \quad f_{i}(i)=0 \text { at } i=0,-1,-2, \cdots,-r+1 \text {, } \\
& \text { (b) } \quad f_{i}(i)+p(i-j)=0 \text { at } i=n+1, n+2, \cdots, n+r \text {. }
\end{aligned}
$$

Then the $n$-vector $v_{i}$ with components

$$
\begin{aligned}
v_{i}(i) & =f_{j}(i), & & i \leqq j, \\
& =f_{i}(i)+p(i-j), & & i \geqq j,
\end{aligned}
$$

is such that $v_{i}(i)=0$ for $i=0,-1,-2, \cdots,-r+1$ and $i=n+1, n+2, \cdots$, $n+r$, due to (6). Hence, the components of $A v_{i}$ are precisely $\delta^{2 r} v_{i}(i)$ for $i=1(1) n$.

But $v_{j}(i)$ agrees with the $(2 r-1)$ st degree polynomial $f_{i}(i)$, at the integer values of $i$ in $i \leqq j+r-1$ (using (7) and the fact that $p(i-j)$ vanishes at $i=j+1$, $j+2, \cdots, j+r-1)$, hence, $\delta^{2 r} v_{j}(i)=0$ at $i=1,2, \cdots, j-1$.

Similarly, $v_{j}(i)$ agrees with the $(2 r-1)$ st degree polynomial $f_{i}(i)+p(i-j)$ at the integer values of $i$ in $i \geqq j-r+1$, so that $\delta^{2 r} v_{i}(i)=0$ at $i=j+$ $1, j+2, \cdots, n$. Indeed, only the $j$ th component of $A v_{i}$ is nonzero. We have $\delta^{2 r} v_{i}(j)=$ $\left[\delta^{2 r} f_{i}(j)\right]+p(r)=1$, since $\delta^{2 r} f_{i}=0$ and $p(r)=1$. We conclude that $v_{i}$ is precisely the $j$ th column of $A^{-1}$.

We summarize in

THEOREM 5. If $p$ and $f$ are defined as in (5) and (6), then 


$$
A^{-1}(2 r+1, n)=\left\{\bar{a}_{i j}\right\}
$$

where

$$
\begin{aligned}
\bar{a}_{i j} & =f_{j}(i), & & i \leqq j, \\
& =f_{j}(i)+p(i-j), & & i \geqq j .
\end{aligned}
$$

In the above theorem, we note that the columns of $A^{-1}$ are pairs of polynomials, in $i$, of degree $2 r-1$, joined at the diagonal. Because of symmetry, the rows of $A^{-1}$ have the same property (regarded as polynomials in $j$ ). Further, since $A$ is centrosymmetric, then $A^{-1}$ is centrosymmetric.

If

$$
L_{r}(i)=i(i+1)(i+2) \cdots(i+r-1),
$$

then the procedure described in Theorem 5 leads to

$$
\begin{aligned}
& \left\{A^{-1}(2 r+1, n)\right\}_{i i}=-\frac{(n+1-i)(n+2-i) \cdots(n+r-i)}{(r-1) !(2 r-1) !} \\
& \cdot \sum_{k=n}^{r-1}\left[(-1)^{k}\left(\begin{array}{c}
r-1 \\
k
\end{array}\right)\left[\frac{L_{r}(i)}{i+k}\right] \frac{(j+k+r-1) !}{(j+k-r) !} \frac{(n+k) !}{(n+k+r) !}\right]
\end{aligned}
$$

for $i \geqq j$.

The first few inverses are given by (for $i \geqq j$ )

$$
\begin{aligned}
\left\{A^{-1}(3, n)\right\}_{i j}= & -\frac{(n+1-i) j}{n+1}, \\
\left\{A^{-1}(5, n)\right\}_{i j}= & -\frac{(n+1-i)(n+2-i) j(j+1)}{1 ! 3 !(n+1)(n+2)(n+3)} \\
& \cdot[(i+1)(j-1)(n+3)-i(j+2)(n+1)], \\
\left\{A^{-1}(7, n)\right\}_{i j}= & -\frac{(n+1-i)(n+2-i)(n+3-i) j(j+1)(j+2)}{2 ! 5 !(n+1)(n+2)(n+3)(n+4)(n+5)} \\
& \cdot[(i+1)(i+2)(j-2)(j-1)(n+4)(n+5) \\
& -2 i(i+2)(j-1)(j+3)(n+1)(n+5) \\
& \quad+i(i+1)(j+3)(j+4)(n+1)(n+2)] .
\end{aligned}
$$

Reference to Lemma 5 provides a series of interesting identities.

Although the problem of determining the eigenvalues and eigenvectors of $A(2 r+1, n)$ is far from solved (except for $r=1)$, we might mention a few relevant facts.

For matrices of even order, we can write

$$
A(2 r+1,2 n)=\left[\begin{array}{cc}
A(2 r+1, n) & B_{n} \\
\ldots \ldots \ldots \ldots \ldots \ldots \ldots \ldots \ldots & \vdots \\
B_{n}^{r} \quad \vdots A(2 r+1, n)
\end{array}\right]
$$


If $P_{n}$ is the matrix whose 1st column is $\hat{e}_{n}$, and whose $j$ th column $(j=2(1) n)$ is $\hat{e}_{i-1}$, then, the characteristic polynomial associated with $A(2 r+1,2 n)$ can be factored, as described in [6]:

$$
\begin{aligned}
\operatorname{det}|A(2 r+1,2 n)-\lambda I| & \\
& =\operatorname{det}\left|A(2 r+1, n)+B_{n} P_{n}-\lambda I\right| \operatorname{det}\left|A(2 r+1, n)-B_{n} P_{n}-\lambda I\right| .
\end{aligned}
$$

For matrices of odd order, we can write

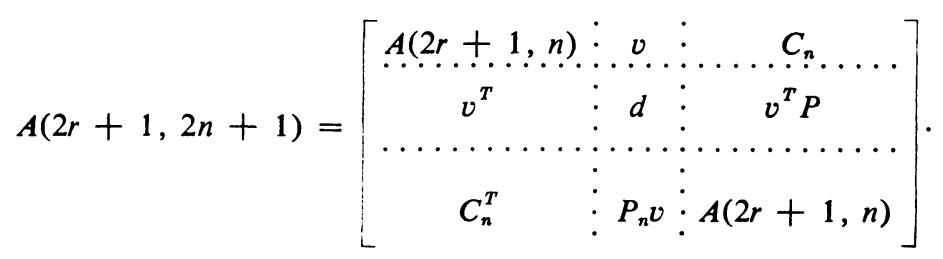

The characteristic polynomial now has the factorized form [6]:

$$
\operatorname{det}|A(2 r+1,2 n+1)-\lambda I|=K(\lambda) \operatorname{det}\left|A(2 r+1, n)-C_{n} P_{n}-\lambda I\right| \text {, }
$$

where $K(\lambda)$ is the characteristic polynomial of

$$
\left[\begin{array}{c}
A(2 r+1, n) \vdots \\
\vdots \\
+C_{n} P_{n} \ldots \\
2 v^{T} \\
\vdots
\end{array}\right]
$$

The above factorization is a result of the centrosymmetry of $A(2 r+1, n)$. This symmetry is reflected in the eigenvectors. We show this as follows.

With $P_{n}$ defined as above, and $A$ used to denote $A(2 r+1, n)$, then $P_{n} A=A P_{n}$ (a result of the centrosymmetry of $A$ ). But, if $x$ is an eigenvector of $A$ with eigenvalue $\lambda$, so $A x=\lambda x$, then $P_{n} A x=A P_{n} x=\lambda P_{n} x$. Hence, $P_{n} x$ is also an eigenvector of $A$, with the same eigenvalue $\lambda$. Consequently, $P_{n} x=\alpha x$ for some scalar $\alpha$ (since all eigenvectors of $A$ which belong to the same eigenvalue are scalar multiples of each other). Thus, $x$ is also an eigenvector of $P_{n}$. But all eigenvectors of $P_{n}$ have either even or odd symmetry about the midpoint. That is,

$$
\begin{array}{ll}
\text { either } & x(n-i+1)=x(i), \quad i=1(1) n . \\
\text { or } \quad & x(n-i+1)=-x(i),
\end{array}
$$

We state the above result in

Lemma 6. All eigenvectors of $A(2 r+1, n)$ satisfy (7).

That is, eigenvectors of $A(2 r+1,2 n)$ have the form

$$
\left[\begin{array}{c}
v \\
\ldots \ldots \\
\pm P_{n} v
\end{array}\right] .
$$

Further, eigenvectors of $A(2 r+1,2 n+1)$ have the form: 


$$
\left|\begin{array}{c|c}
v \\
\cdots \cdots \\
0 \\
\cdots \cdots \\
-P_{n} v
\end{array}\right| \text { or }\left|\begin{array}{c}
v \\
\cdots \\
\cdots \\
P_{n} v
\end{array}\right| \text {. }
$$

The ease with which the properties of this special class of matrices has been analysed suggests that further useful work remains to be done on the determination of corresponding results for block matrices with special structures.

Department of Computer Science

University of Manitoba

Winnipeg 19, Manitoba. Canada

Department of Applied Mathematics

University of Waterloo

Waterloo, Ontario Canada

1. R. G. Stanton \& D. A. Sprotr, "Some finite inversion formulae," Math. Gazette, v. 46, 1962, pp. 197-202.

2. R. S. VARGA, Matrix Iterative Analysis, Prentice-Hall, Englewood Cliffs, N.J., 1962, pp. 161-208. MR 28 \#1725.

3. L. Fox (Editor), Numerical Solution of Ordinary and Partial Differential Equations, Pergamon Press, Oxford; Addison-Wesley, Reading, Mass., 1962. MR 26 \#4488.

4. D. E. RutherFoRD, "Some continuant determinants arising in physics and chemistry.

II," Proc. Roy. Soc. Edinburgh Sect. A, v. 63, 1952, pp. 232-241. MR 15, 495.

5. J. Riordan, Combinatorial Identities, Wiley, New York, 1968, p. 8. MR 38 \#53.

6. A. C. AITKEN, Determinants and Matrices, Oliver \& Boyd, London, 1936, p. 124. 